\title{
Case Report of Syncope during a Single Pulse Transcranial Magnetic Stimulation Experiment in a Healthy Adult Participant
}

\author{
Trisha M. Kesar ${ }^{\star}$, Haley S. McDonald, Steven P. Eicholtz, and Michael R. Borich \\ Department of Rehabilitation Medicine, Division of Physical Therapy, Emory University, Atlanta, \\ Georgia, USA
}

\section{Dear Editor}

We report the following TMS-related syncope spell during a single-pulse transcranial magnetic stimulation (TMS) experiment. The participant was a 25 -year old male. During the consenting process, the participant completed a TMS screening form based on published TMS safety guidelines [1] and reported no previous history of seizures, epilepsy, medical conditions, and no current medications. On the day of the event, the participant had no additional risk factors such as change in sleep pattern, sleep deprivation, change in medication, occult drug use, or high doses of caffeine.

Testing was initiated at $9 \mathrm{am}$. Single TMS pulses were delivered over the inter-hemispheric fissure overlying the lower extremity motor cortex representation using a custom batwing coil (Magstim, UK) connected to a Magstim $200^{2}$ monophasic stimulator at a frequency of $<0.2 \mathrm{~Hz}$ at $30-40 \% \mathrm{MSO}$. The participant had not undergone TMS previously. The participant was seated on a chair with his back and thighs supported and both feet on the floor. During hotspot determination, ankle weights were placed over the right foot for stabilization. The setting was a research lab within a rehabilitation hospital. The incident happened after approximately 40 pulses during determination of the motor hotspot of the right tibialis anterior, $\sim 45$ minutes after start of the experiment. At this point, the participant mentioned feeling a tingling sensation in his right lower limb in response to TMS. After about a minute, the participant stated "I feel dizzy, I'm going to pass out" and immediately, he lost consciousness. The TMS operator noted that the participant's neck was somewhat rigid as his head turned to the left and fell back toward the chair, with his eyes closed. The TMS operator stabilized the participant's head and other team members assisted with getting him in a comfortable, seated, semi-reclined position with his head and upper back supported. We were unable to transition the participant to a lying down position due to limited space and equipment surrounding the testing area. During the episode of syncope, there was substantial pallor evident in the participant's face. After about 30-seconds, color returned to his face and neck with a bright pink coloration of the skin. One study team member also noted 2-3 jerky tonic movements from the participant's upper and lower limbs.

"Corresponding author. Emory Rehabilitation Hospital, 1441, Clifton Rd NE, Atlanta, Georgia 30322, USA, Tel.: +1 4047125803; fax: 404-712-4130. tkesar@emory.edu (T.M. Kesar). 
About 45 seconds following episode onset, the participant regained consciousness, responded when spoken to, and was able to identify correctly where he was (oriented to space and time). Upon regaining consciousness, the participant appeared to have good recollection of the events before the loss of consciousness. He stated that he felt tired, dizzy, and nauseous, and could not see clearly. During the 20-minute period following the episode, the participant appeared anxious and overwhelmed, and proceeded to vomit twice. The experimenters continued to monitor the participant, communicate with him, and kept him in a safe, stable, supported position. The TMS operator and another team member were certified in cardiopulmonary resuscitation. Within 45-minutes following the episode, a physician from the hospital checked on the participant, and the study team continued to communicate with and monitor the participant within the laboratory. During or after the episode of syncope, the participant did not have urinary, fecal incontinence, postictal confusion, tongue biting, or other physical trauma. The episode of syncope self-terminated. As a precautionary measure, the participant was advised to visit the emergency room for follow-up evaluation.

A member of the study team accompanied the participant to the emergency room, where cardiovascular and neurologic screens were performed by the emergency care team, approximately 120 minutes after the event. At the emergency room, the participant's blood pressure, heart rate, and blood work were within normal limits. The participant also received a CT scan, which was confirmed to be normal by a radiologist. Electroencephalographic assessment was not performed. Upon discharge, the participant had no residual symptoms, but continued to qualitatively report feeling exhausted for several hours after discharge. The study team followed up with the participant 24 hours and 30 days after the incident and he reported no residual symptoms or negative sequelae. During the hours following the incident, detailed discussion with the participant revealed information about his past medical history that may or may not be pertinent to the syncope. First, although the participant had no recent history of loss of consciousness, he reported (upon discussion after the incident) that he had once experienced light-headedness and fainting when donating blood. Second, the participant reported minor closed head injuries over 9 years ago, during participation in high-impact sport, which he had not remembered or considered relevant during the TMS screening process. Third, although there was no recent episode, the participant occasionally experienced headaches or migraines in the past. The patient was withdrawn from the study and did not undergo further TMS testing.

The clinical diagnosis of this event was TMS-related syncope. The specific reasons for favoring this choice versus the differential diagnoses of TMS-related seizure were past history of syncope during venipuncture, very brief duration of the loss of consciousness ( 45-seconds), absence of fecal or urinary continence, rapid regaining of consciousness and orientation after the episode, lack of confusion or neurologic sequelae following the event, lack of convulsions, and previous published cases reporting similar episodes and symptoms [2-6].

As a cautionary measure, following this event, the study exclusion criteria were modified to exclude individuals with history of loss of consciousness in the past 12 months.

Additionally, we now emphasize that participants carefully report any history of concussion 
(suspected or formally diagnosed), as well as any residual symptoms following the concussion, regardless of time since occurrence. Although rare, similar TMS-related episodes of vasovagal syncope in healthy individuals [2,3] and children with stroke [4] with no other risk factors for TMS have been reported during single-pulse TMS in recent literature, and in published TMS safety guidelines [1]. Kirton and colleagues report that it is unsurprising that anxiety during exposure to TMS, a novel and mildly noxious stimulus, precipitates syncope, and suggest screening for risk factors such as previous history of syncope, stress, and hunger, to minimize the risk of such incidents [4]. Rare cases of seizure have also been reported in healthy individuals during single-pulse TMS [7]. These cases of TMS-related convulsive syncope and seizures, albeit unusual, suggest the need to comprehensively screen, document, and plan for the management of these episodes during experiments involving TMS. Previous safety guidelines emphasize the need for adequate space in the testing area, and a place in the laboratory for the subject to lie down in case of an episode of syncope or seizures [1]. Additionally, the fact that the participant did not remember (or considered irrelevant) the history of minor head injury from over 9 years ago, in conjunction with the expected prevalence of minor and often undiagnosed concussions in young individuals, emphasizes the potential need for increased scrutiny during the TMS screening process for able-bodied control participants. Additionally, risk factors predisposing to TMS-related syncope or seizures should continue to be carefully monitored and further characterized to minimize risk of future incidence.

\section{Acknowledgments}

Funding sources: NIH NICHD K01 HD079584 awarded to Dr. Kesar, and NIH K12 HD055931 awarded to Dr. Borich.

\section{References}

1. Rossi S, Hallett M, Rossini PM, Pascual-Leone A. Safety, ethical considerations, and application guidelines for the use of transcranial magnetic stimulation in clinical practice and research. Clin Neurophysiol. 2009; 120(12):2008-39. [PubMed: 19833552]

2. Gillick BT, Rich T, Chen M, Meekins GD. Case report of vasovagal syncope associated with single pulse transcranial magnetic stimulation in a healthy adult participant. BMC Neurol. 2015; 15:248. [PubMed: 26627895]

3. Hadar AA, Makris S, Yarrow K. Single-pulse TMS related syncopal spell in a healthy subject. Brain Stimul. 2012; 5(4):652-3. [PubMed: 21930450]

4. Kirton A, Deveber G, Gunraj C, Chen R. Neurocardiogenic syncope complicating pediatric transcranial magnetic stimulation. Pediatr Neurol. 2008; 39(3):196-7. [PubMed: 18725065]

5. Epstein CM. Seizure or convulsive syncope during 1-Hz rTMS? Clin Neurophysiol. 2006; 117(11): 2566-7. author reply 2567-8. [PubMed: 16996304]

6. Romme JJ, van Dijk N, Boer KR, Bossuyt PM, Wieling W, Reitsma JB. Diagnosing vasovagal syncope based on quantitative history-taking: validation of the Calgary Syncope Symptom Score. Eur Heart J. 2009; 30(23):2888-96. [PubMed: 19687157]

7. Kratz O, Studer P, Barth W, Wangler S, Hoegl T, Heinrich H, et al. Seizure in a nonpredisposed individual induced by single-pulse transcranial magnetic stimulation. J ECT. 2011; 27(1):48-50. [PubMed: 20351571] 